\title{
Differential expression of Lp-PLA2 in obesity and type 2 diabetes and the influence of lipids
}

\author{
Laura Jackisch $^{1}$ - Warunee Kumsaiyai ${ }^{2} \cdot$ Jonathan D. Moore ${ }^{3} \cdot$ Nasser Al-Daghri ${ }^{4,5} \cdot$ Ioannis Kyrou ${ }^{1,6}$. \\ Thomas M. Barber ${ }^{1,7}$ • Harpal Randeva ${ }^{1}$. Sudhesh Kumar ${ }^{1}$ - Gyanendra Tripathi ${ }^{1,8}$ • Philip G. McTernan ${ }^{1,9}$
}

Received: 27 July 2017 / Accepted: 3 January 2018 / Published online: 9 February 2018

(C) The Author(s) 2018. This article is an open access publication

\begin{abstract}
Aims/hypothesis Lipoprotein-associated phospholipase A2 (Lp-PLA2) is a circulatory macrophage-derived factor that increases with obesity and leads to a higher risk of cardiovascular disease (CVD). Despite this, its role in adipose tissue and the adipocyte is unknown. Therefore, the aims of this study were to clarify the expression of Lp-PLA2 in relation to different adipose tissue depots and type 2 diabetes, and ascertain whether markers of obesity and type 2 diabetes correlate with circulating Lp-PLA2. A final aim was to evaluate the effect of cholesterol on cellular Lp-PLA2 in an in vitro adipocyte model.

Methods Analysis of anthropometric and biochemical variables from a cohort of lean (age $44.4 \pm 6.2$ years; BMI $22.15 \pm 1.8 \mathrm{~kg}$ / $\mathrm{m}^{2}, n=23$ ), overweight (age $45.4 \pm 12.3$ years; BMI $26.99 \pm 1.5 \mathrm{~kg} / \mathrm{m}^{2}, n=24$ ), obese (age $49.0 \pm 9.1$ years; BMI $33.74 \pm 3.3 \mathrm{~kg}$ / $\mathrm{m}^{2}, n=32$ ) and type 2 diabetic women (age $53.0 \pm 6.13$ years; BMI $35.08 \pm 8.6 \mathrm{~kg} / \mathrm{m}^{2}, n=35$ ), as part of an ethically approved study. Gene and protein expression of PLA2 and its isoforms were assessed in adipose tissue samples, with serum analysis undertaken to assess circulating Lp-PLA2 and its association with cardiometabolic risk markers. A human adipocyte cell model, Chub-S7, was used to address the intracellular change in Lp-PLA2 in adipocytes.

Results Lp-PLA2 and calcium-independent PLA2 (iPLA2) isoforms were altered by adiposity, as shown by microarray analysis $(p<0.05)$. Type 2 diabetes status was also observed to significantly alter gene and protein levels of Lp-PLA2 in abdominal subcutaneous $(\mathrm{AbdSc})(p<0.01)$, but not omental, adipose tissue. Furthermore, multivariate stepwise regression analysis of circulating Lp-PLA2 and metabolic markers revealed that the greatest predictor of Lp-PLA2 in non-diabetic individuals was LDL-cholesterol $(p=0.004)$. Additionally, in people with type 2 diabetes, oxidised LDL (oxLDL), triacylglycerols and HDLcholesterol appeared important predictors, accounting for $59.7 \%$ of the variance $(p<0.001)$. Subsequent in vitro studies determined human adipocytes to be a source of Lp-PLA2, as confirmed by mRNA expression, protein levels and immunochemistry. Further in vitro experiments revealed that treatment with LDL-cholesterol or oxLDL resulted in significant upregulation of LpPLA2, while inhibition of Lp-PLA2 reduced oxLDL production by $19.8 \%(p<0.05)$.
\end{abstract}

Electronic supplementary material The online version of this article (https://doi.org/10.1007/s00125-018-4558-6) contains peer-reviewed but unedited supplementary material, which is available to authorised users.

Gyanendra Tripathi

g.tripathi@westminster.ac.uk

Philip G. McTernan

philip.mcternan@ntu.ac.uk

1 Division of Biomedical Sciences, Warwick Medical School, University of Warwick, Coventry, UK

2 Department of Medical Technology, Chiang Mai University, Chiang Mai, Thailand

3 Warwick Systems Biology Centre, University of Warwick, Coventry, UK

4 Biomarkers Research Program, Biochemistry Department, King Saud University, Riyadh, Saudi Arabia
5 Prince Mutaib Chair for Biomarkers of Osteoporosis, Biochemistry Department, King Saud University, Riyadh, Saudi Arabia

6 Aston Medical Research Institute, Aston Medical School, Aston University, Birmingham, UK

7 Human Metabolism Research Unit, Warwickshire Institute for the Study of Diabetes, University Hospitals Coventry and Warwickshire NHS Trust, Coventry, UK

8 Department of Biomedical Sciences, University of Westminster, 115 New Cavendish Street, London W1W 6UW, UK

9 College of Science and Technology, Department of Biosciences, Nottingham Trent University, Clifton, Nottingham NG1 8NS, UK 


\section{Research in context}

\section{What is already known about this subject?}

- Increasing serum lipoprotein-associated phospholipase A2 (LP-PLA2) levels are associated with obesity and cardiovascular disease, in addition to an elevated risk of mortality and morbidity over time

- PLA2 is classified into three groups: calcium-dependent PLA2 (CPLA2), calcium-independent PLA2 (iPLA2) and LpPLA2, of which iPLA2 and CPLA2 are known to contribute to inflammation through the production of lipid mediators

- Lp-PLA2, which has been documented in monocytes, macrophages and T lymphocytes, catalyses the hydrolysis of oxLDL, catalysing the proinflammatory mediators lysophosphatidylcholine and oxidised fatty acids

\section{What is the key question?}

- Are PLA2 levels altered by obesity, type 2 diabetes and differing fat depots, and how is this influenced by lipids in human adipocytes?

\section{What are the new findings?}

- PLA2 isoforms are differentially expressed according to depot specificity, adiposity and type 2 diabetes status

- Circulating Lp-PLA2 levels are influenced by unfavourable lipid profiles in individuals with type 2 diabetes

- In addition to macrophage-induced Lp-PLA2, the adipocyte itself represents a further active site of Lp-PLA2

\section{How might this impact on clinical practice in the foreseeable future?}

- This study highlights the importance of ensuring systemic lipids profiles are actively managed to reduce further complications that may result from the inflammatory response of the adipocyte

Conclusions/interpretation Our study suggests adipose tissue and adipocytes are active sources of Lp-PLA2, with differential regulation by fat depot and metabolic state. Moreover, levels of circulating Lp-PLA2 appear to be influenced by unfavourable lipid profiles in type 2 diabetes, which may occur in part through regulation of LDL-cholesterol and oxLDL metabolism in adipocytes.

Keywords Adipose tissue · Inflammation · Lipids · Lipoprotein-associated phospholipase A2 · Low density lipoprotein · Obesity · Oxidised low density lipoprotein · Type 2 diabetes

$\begin{array}{ll}\text { Abbreviations } \\ \text { AbdSc } & \text { Abdominal subcutaneous } \\ \text { cPLA2 } & \text { Calcium-dependent PLA2 } \\ \text { CVD } & \text { Cardiovascular disease } \\ \text { iPLA2 } & \text { Calcium-independent PLA2 } \\ \text { Lp-PLA2 } & \text { Lipoprotein-associated PLA2 } \\ \text { MAPK } & \text { Mitogen-activated protein kinase } \\ \text { oxLDL } & \text { Oxidised LDL } \\ \text { PLA2 } & \text { Phospholipase A2 } \\ \text { qPCR } & \text { Quantitative PCR } \\ \text { SVF } & \text { Stromal vascular fraction }\end{array}$

\section{Introduction}

Lipoprotein-associated phospholipase A2 (Lp-PLA2) is a member of the phospholipase A2 superfamily of enzymes, which promote the formation of oxidised LDL (oxLDL), a producer of proinflammatory mediators such as lysophosphatidylcholine and oxidised fatty acids (enhanced in states of metabolic disease) [1,2]. Prior studies highlight that circulating Lp-PLA2 directly increases arterial inflammation, while cytosolic calcium-dependent PLA2 (cPLA2) and calcium-independent PLA2 (iPLA2) appear to contribute to inflammation via immunological cells [3]. To date, much attention has focused on changes in circulating Lp-PLA2 and disease (arising from epidemiological studies), and has highlighted that Lp-PLA2 is upregulated in conditions of obesity, inflammation and cardiovascular disease (CVD) [4-7].

Macrophage-derived Lp-PLA2 has been shown to promote the instability of vulnerable atherosclerotic plaques, increasing the risk of coronary events; Lp-PLA2 inhibitors have been shown to reduce the frequency of these occurrences [8]. Despite this clear connection with CVD, few studies have explored the impact of circulating Lp-PLA2 in people with type 2 diabetes, which is often considered to precede CVD. The literature suggests that individuals with type 2 diabetes have raised circulating Lp-PLA2 levels, and that these are positively correlated with adiposity and cardiometabolic risk 
factors $[9,10]$. While these studies indicate that increased LpPLA2 with adiposity arises as a result of macrophages, the importance of human adipocytes in Lp-PLA2 metabolism remains unclear. This is despite the knowledge that adipocytes possess many overlapping immune cell characteristics, including the production of proinflammatory biomarkers $[11,12]$.

This study sought to investigate the role of Lp-PLA2 in the adipocyte and the impact of various metabolic states within adipose tissue on Lp-PLA2 expression. Our aims were to: (1) characterise PLA2 gene expression and its related isoforms in adipose tissue; (2) determine the depot-specific expression within adipose tissue and the impact of obesity and type 2 diabetes; (3) define how cardiometabolic risk factors are associated with circulating Lp-PLA2 levels within different metabolic states; and (4) evaluate the molecular impact of LDLcholesterol and oxLDL on Lp-PLA2 expression within adipocytes.

\section{Methods}

Participants Ethical approval was obtained from the Local Research Ethics Committee and all participants gave written and informed consent. For this study, 114 women undergoing elective abdominal surgery were recruited. The cohort consisted of lean (age $44.4 \pm 6.2$ years; BMI $22.15 \pm 1.8 \mathrm{~kg} / \mathrm{m}^{2}, n=23$ ), overweight (age $45.4 \pm$ 12.3 years; BMI $26.99 \pm 1.5 \mathrm{~kg} / \mathrm{m}^{2}, n=24$ ), obese (age $49.0 \pm 9.1$ years; BMI $33.74 \pm 3.3 \mathrm{~kg} / \mathrm{m}^{2}, n=32$ ) and women with type 2 diabetes (age $53.0 \pm 6.13$ years; BMI $\left.35.08 \pm 8.6 \mathrm{~kg} / \mathrm{m}^{2}, n=35\right)$. Detailed medical drug histories were taken and those participants with cancer, thyroid disorders or taking steroids or medication considered to alter inflammatory status, including thiazolidinediones, were excluded.

Serum and tissue collection Venous blood samples were taken after an 8-10 h overnight fast. Adipose tissue was obtained by needle biopsy and then flash frozen and/or used for in vitro studies.

In vivo assessment of biochemical profile Fasting blood samples were collected from participating volunteers. Lipid profiles and fasting plasma glucose were determined using routine laboratory methods at the University Hospitals Coventry and Warwickshire NHS Trust. In brief, the routine blood tests included glucose and a standard lipidaemic/cholesterol profile (triacylglycerols, HDL-cholesterol and LDL-cholesterol), as noted in Table 1. OxLDL and Lp-PLA2 were measured by ELISA (oxLDL ELISA kit, Mercodia, Uppsala, Sweden; intra-assay $\% \mathrm{CV}=6.4$; inter-assay $\% \mathrm{CV}=7.4$; and Human PLA2 G7/PAF-AH/Lp-PLA2 Quantikine ELISA, R\&D Systems, Abingdon, UK; intra-assay $\% \mathrm{CV}=6.8$, inter-assay
$\% \mathrm{CV}=9.6$; respectively). Insulin measurements were performed using a solid-phase enzyme amplified sensitivity multiplex immunoassay (Millipore, Watford, UK), and glucose was measured by a glucose oxidase method (YSL 200 STAT plus, Yellow Springs Instruments, Yellow Springs, OH, USA).

Analysis of circulating endotoxins Serum endotoxin was analysed using the QCL-1000 LAL endpoint assay (Lonza, Allendale, NJ, USA). The assay, and the values given by the manufacturer for intra-assay \%CV $(3.9 \pm 0.46 \%)$ and interassay $\% \mathrm{CV}(9.6 \pm 0.75 \%)$, have been validated in our laboratory, as detailed previously $[13,14]$.

Isolation of pre-adipocytes, stromal vascular fraction and mature adipocytes Abdominal subcutaneous (AbdSc) adipose tissue was digested as previously described to isolate stromal vascular fraction (SVF), pre-adipocytes and mature adipocytes [15]. In short, adipose tissue was incubated with collagenase for $30 \mathrm{~min}$, the digest was then filtered through a cotton mesh and centrifuged. Differential centrifugation resulted in floating mature adipocytes and pellets of SVF. The preadipocytes were cultured, while RNA was extracted directly from SVF and mature adipocytes.

Protein determination and western blot analysis A subgroup of paired human AbdSc and omental adipose tissue biopsies from participants who were lean (age $43.6 \pm 6.2$ years; BMI $22.5 \pm 2.2 \mathrm{~kg} / \mathrm{m}^{2} ; n=9$ ), overweight (age $47.5 \pm$ 11.5 years; BMI $27.4 \pm 1.5 \mathrm{~kg} / \mathrm{m}^{2} ; n=10$ ) or obese (age $48.1 \pm 8.5$ years; BMI $34.0 \pm 2.9 \mathrm{~kg} / \mathrm{m}^{2} ; n=5$ ) was used for protein analysis. The adipose tissue was homogenised in Phosphosafe extraction buffer (Novagen, Merck, Darmstadt, Germany) and cultured adipocytes were harvested in RIPA buffer (Cell Signaling, Denver, MA, USA) with a cocktail of protease inhibitors, to extract total protein. Protein concentrations were measured using the BioRad Detergent Compatible protein assay kit (Bio-Rad, San Diego, CA, USA) [16]. Western blotting was performed as described elsewhere [17], and protein levels of cPLA2 (1:100, Cell Signaling), iPLA2 (1:500, Sigma, Poole, UK) and Lp-PLA2 (1:200, R\&D Systems) were assessed with rabbit and goat monoclonal antibodies.

RNA extraction and quantitative PCR RNA was extracted from samples using an RNeasy lipid tissue kit (Qiagen, Manchester, UK) according to the manufacturer's instructions, followed by a DNase digestion step. cDNA was synthesised using reverse transcription reagents (Bioline, London, UK). Quantitative (q)PCR was performed with TaqMan probes $(18 \mathrm{~S}$, Hs03003631 g1; PLA2G7, Hs00173726 m1; PLA2G4, Hs00233352 m1; PLA2G6, Hs00185926 m1; CD68, Hs02836816_g1; CD206 [also known as $M R C 1$ ], Hs00267207_m1; HLA-DRA, Hs00219575_m1; CIITA, 
Table 1 Selected characteristics of the study participants with and without type 2 diabetes

\begin{tabular}{|c|c|c|c|c|}
\hline \multirow[t]{2}{*}{ Characteristic } & \multicolumn{3}{|l|}{ Non-diabetic } & \multirow{2}{*}{$\begin{array}{l}\text { Type } 2 \text { diabetic } \\
(n=35)\end{array}$} \\
\hline & $\begin{array}{l}\text { Lean } \\
(n=23)\end{array}$ & $\begin{array}{l}\text { Overweight } \\
(n=24)\end{array}$ & Obese $(n=32)$ & \\
\hline BMI $\left(\mathrm{kg} / \mathrm{m}^{2}\right)$ & $22.15 \pm 1.8$ & $26.99 \pm 1.5^{* * *}$ & $33.74 \pm 3.2^{* * *}$ & $35.08 \pm 8.6^{* * * *}$ \\
\hline Glucose $(\mathrm{mmol} / \mathrm{l})$ & $4.61 \pm 0.1$ & $4.84 \pm 0.1$ & $5.12 \pm 0.2^{* *}$ & $8.56 \pm 0.4^{* * * *+\dagger}$ \\
\hline HOMA-IR & $0.89 \pm 0.1$ & $1.12 \pm 0.1$ & $2.64 \pm 0.3^{* * * *}$ & $6.21 \pm 0.8^{* * * *+\dagger}$ \\
\hline Cholesterol (mmol/l) & $4.55 \pm 0.2$ & $4.96 \pm 0.2$ & $5.24 \pm 0.2^{*}$ & $4.97 \pm 0.2$ \\
\hline Triacylglycerol (mmol/l) & $0.76 \pm 0.1$ & $0.91 \pm 0.1$ & $1.52 \pm 0.2^{* * * *}$ & $1.44 \pm 0.1^{* * * *}$ \\
\hline LDL-cholesterol (mmol/l) & $2.32 \pm 0.2$ & $2.73 \pm 0.2$ & $3.07 \pm 0.1^{* *}$ & $3.24 \pm 0.2^{* *}$ \\
\hline HDL-cholesterol (mmol/l) & $1.88 \pm 0.1$ & $1.81 \pm 0.1$ & $1.45 \pm 0.1^{* *}$ & $1.24 \pm 0.1^{* * * * \dagger}$ \\
\hline $\begin{array}{l}\text { LDL-cholesterol } \\
\text { /HDL-cholesterol }\end{array}$ & $1.31 \pm 0.5$ & $1.66 \pm 0.2$ & $2.39 \pm 0.2^{* * * *}$ & $2.87 \pm 0.2^{* * * * \dagger}$ \\
\hline Insulin $(\mathrm{pmol} / \mathrm{l})$ & $28.95 \pm 8.0$ & $33.24 \pm 7.6$ & $69.45 \pm 7.6^{* * * *}$ & $91.55 \pm 7.8^{* * * * \dagger}$ \\
\hline Endotoxin (EU/ml) & $2.12 \pm 0.2$ & $2.91 \pm 0.3^{*}$ & $4.39 \pm 0.4^{* * * *}$ & $6.95 \pm 0.3^{* * * *+\dagger}$ \\
\hline OxLDL (U/l) & $39.17 \pm 1.5$ & $41.08 \pm 2.5$ & $51.87 \pm 3.1^{* * * *}$ & $66.88 \pm 4.4^{* * * *+\dagger}$ \\
\hline Lp-PLA2 (pmol/l) & $2.39 \pm 0.14$ & $2.81 \pm 0.21$ & $2.98 \pm 0.19^{*}$ & $2.94 \pm 0.16^{*}$ \\
\hline
\end{tabular}

Data are means \pm SEM, except for BMI which is mean \pm SD

Unpaired $t$ test was used to compare means

$* p<0.05, * * p<0.01$ and $* * * p<0.001$ for lean vs overweight, obese and type 2 diabetic

$\uparrow p<0.05, \uparrow \uparrow p<0.01$ and $\uparrow \uparrow \uparrow p<0.001$ for obese vs type 2 diabetic
Hs00172106_m1; EMR1 [also known as ADGRE1], Hs00173562_m1; Applied Biosystems, Warrington, UK). Transcript abundance was measured with an Applied Biosystems 7500 Real-Time PCR System with TaqMan universal PCR master mix. All reactions were multiplexed with the housekeeping gene $18 \mathrm{~S}$, to normalise qPCR data.

Immunohistochemistry Adipose and placenta tissue samples were incubated with primary polyclonal Lp-PLA2 antibody (R\&D Systems) in a dilution of 1:100. Sections were developed using peroxidase substrate kit VIP (Vector Laboratories, Peterborough, UK) for Lp-PLA2. To demonstrate specific binding, the primary antibody was omitted for negative control for Lp-PLA2 independently.

Microarray analysis RNA from the adipose tissue samples was used for gene expression analysis with the Human Genome U133A plus 2.0 DNA microarrays (Affymetrix, Santa Clara, CA, USA). Preparation of cRNA and hybridisation to DNA microarrays were performed according to standard Affymetrix protocols, as previously described $[18,19]$. PLA2 mRNA expression was investigated using the 219064_AT probe set.

Cell cultures AbdSc pre-adipocytes were grown to confluence in DMEM/F-12 containing 10\% (vol./vol.) fetal bovine serum, $1 \%$ (vol./vol.) penicillin/streptomycin and transferrin $(62.5 \mathrm{pmol} / \mathrm{l})$ at $37^{\circ} \mathrm{C}, 5 \% \mathrm{CO}_{2}$ incubation. For cell differentiation, AbdSc pre-adipocytes were maintained in PromoCell pre-adipocyte differentiation media (PromoCell, Heidelberg, Germany) for $48 \mathrm{~h}$. Subsequently, the cells were maintained in the PromoCell adipocyte nutrition media (PromoCell, Heidelberg, Germany) for 14 days, with the medium changed every 2 days. The differentiated adipocytes were then given a $24 \mathrm{~h}$ wash out in DMEM/F12 supplemented with $0.5 \%$ (wt/ vol.) BSA. Chub-S7 cells, a human AbdSc pre-adipocyte cell line, were grown under the same conditions [20].

The differentiated AbdSc Chub-S7 cells $(n=6)$ were treated with LDL-cholesterol $(67 \mathrm{pmol} / \mathrm{l}) \pm 20 \mu \mathrm{mol} / \mathrm{l}$ of the Lp-PLA2 inhibitor (Darapladib, Cayman Chemical, Ann Arbor, Michigan, USA) or oxLDL (43 pmol/1; Kalen Biomedical, Germantown, MD, USA) for 3, 6, 24 and $48 \mathrm{~h}$. PBS containing $0.34 \mathrm{mmol} / \mathrm{l}$ EDTA was used as the control. Experiments were conducted with six technical replicates per treatment.

Statistical analysis For microarray data analysis, one-way ANOVA was performed for each selected PLA2 gene. Significance of mRNA expression and protein levels in different adiposity, fat depot and type 2 diabetes status data were analysed with paired $t$ tests. For the cell culture, treatments were compared using two-way ANOVA. All quantitative variables are shown as the mean \pm SEM, unless otherwise stated.

Determination of correlations in gene expression analysis was performed using Pearson's correlation coefficient. Spearman's rank correlation coefficient was used to determine correlations for serum Lp-PLA2 and metabolic markers because of the non-parametric distribution. Furthermore, multivariate stepwise regression was applied to calculate predictors of systemic Lp-PLA2 and Bonferroni correction was used to adjust the $p$ value to 0.0045 . Power analyses to determine sample size were carried out using $G^{*}$ Power version 3.1.9.2 
(G*power, Düsseldorf, Germany). Analyses and graphing were performed using SPSS version 18.0 for Windows (SPSS, Chicago, Illinois, USA) and GraphPad Prism version 7.01 (GraphPad, La Jolla, CA, USA), respectively. Levels of statistical significance were set at $* p<0.05, * * p<0.01$ and $* * * p<0.001$, unless otherwise stated.

\section{Results}

PLA2 microarray expression profile in adipose tissue A microarray approach containing 20 probes was used to obtain a comprehensive picture of genes differentially expressed in AbdSc and omental adipose tissue from lean and obese participants. The probes corresponded to genes encoding for four isoenzymes in the platelet-activating factor acetylhydrolase family (PLA2G7, PAFAH2, PAFAH1B1 and PAFAH1B2), three isoenzymes in the cPLA2 family (PLA2G4A, PLA2G4C and $P L A 2 G 4 D)$, four isoenzymes in the iPLA2 family (PLA2G6, PNPLA2, PNPLA4 and PNPLA5) and nine isoenzymes in the secretory PLA2 family (PLA2G1B, PLA2G2A, PLA2G2D, PLA2G2E, PLA2G2F, PLA2G5, PLA2G10, PLA2G12A and $P L A 2 G 12 B)$. Increased expression $(p<0.05)$ was noted for PLA2G7 (encoding Lp-PLA2), while PLA2G6 (encoding iPLA2) showed downregulation $(p<0.05)$ in AbdSc adipose tissue taken from obese individuals compared with lean individuals (Fig. 1a). Our raw array data are available online at Open Science Network (https://osf.io/s6rw3/).

PLA2 family mRNA and protein levels in adipose tissue To further characterise PLA2 in relation to adiposity, we investigated PLA2G7, PLA2G6 and PLA2G4 (encoding cPLA2) expression in subcutaneous and omental adipose tissue sampled from lean, overweight and obese individuals.

The level of PLA2G7 mRNA was significantly higher in AbdSc than omental adipose tissue in obese participants $(p<0.05)$; no difference was observed between the other groups (Fig. 2a). PLA2G7 gene expression was raised in adipose tissue samples from overweight or obese individuals compared with lean individuals, but the difference was not significant (Fig. 2a). Analysis of Lp-PLA2 protein indicated that increasing adiposity alone did not increase Lp-PLA2 levels in adipose tissue (Fig. 2b).

Analysis of PLA2G4 gene expression demonstrated that PLA2G4 was expressed preferentially in OM adipose tissue depots of lean individuals rather than AbdSc adipose tissue $(p<0.001$; Fig. 2c). Overweight and obese individuals had higher levels of PLA2G4 gene expression in the AbdSc adipose tissue depot compared with their lean counterparts $(p<0.05)$. Interestingly, in the omental adipose tissue depot, PLA2G4 mRNA expression was significantly decreased in the obese participants $(p<0.001$; Fig. 2 c). Subsequent cPLA2 protein analysis confirmed the gene expression findings for AbdSc and omental adipose tissue (Fig. 2d).

Next, we investigated PLA2G6 gene expression between paired AbdSc and omental adipose tissue and no effect of adiposity was observed (Fig. 2e). Protein analysis, however, revealed that levels of iPLA2 were higher in omental than AbdSc adipose tissue in lean and overweight participants ( $p<0.05$; Fig. 2f). Taken together, these results indicate that depot specificity and differing levels of adiposity affect PLA2 expression in adipose tissue.

Influence of type 2 diabetes status on Lp-PLA2 Given that PLA2 levels were markedly altered by adiposity, we investigated the influence of type 2 diabetes status. Analysis of PLA2G7 gene expression in AbdSc adipose tissue depots from lean, obese and type 2 diabetic individuals showed a significant increase in diabetic adipose tissue compared with lean adipose tissue $(p<0.01$; Fig. 3a). Consistent with these findings, protein analysis demonstrated increased levels of Lp-PLA2 in individuals with type 2 diabetes; however, this increase was more modest and did not reach statistical significance (Fig. 3b). There were no significant differences in AbdSc adipose tissue mRNA and protein levels of cPLA2 and iPLA2 between the non-diabetic and type 2 diabetic groups (data not shown). Thus, type 2 diabetes status appeared to be associated with an upregulation of Lp-PLA2 in adipose tissue.

Analysis of Lp-PLA2 and macrophage markers in adipocytes and adipose tissue In view of the changes of Lp-PLA2 in adipose tissue, our study further sought to investigate the source of Lp-PLA2 in adipose tissue. Using immunohistochemical staining, we observed the expression of Lp-PLA2, as denoted by the brown staining observed around each cell, in mature adipocytes from lean non-diabetic individuals (Fig. 4a). Placental tissue was used as a positive control for Lp-PLA2 staining, with positive brown staining also shown, in addition to negative staining noted in both tissue sections (Fig. 4a).

Consistent with these findings, protein analysis demonstrated the presence of Lp-PLA2 in a pure human adipocyte cell line, Chub-S7, pre- and post-differentiation (Fig. 4b). Analysis of LpPLA2 content in cultured primary human adipocytes from AbdSc adipose tissue also revealed the presence of Lp-PLA2 in both pre-adipocytes and mature adipocytes.

Given that macrophages are known to express Lp-PLA2 in adipose tissue, we determined the expression of macrophage markers in mature adipocyte samples. No differences were observed in PLA2G7 mRNA expression between mature adipocytes and SVF isolated from adipose tissue after collagenase digestion (Fig. 4c). Mature adipocytes had reduced levels of the macrophage markers CD68, CD206, HLA-DRA, CIITA and EMR1 in comparison with SVF (Fig. 4c). Additionally, no significant correlations were identified between the gene 
Fig. 1 Microarray data analysis of the PLA2 gene family. The data are represented as a ratio between intensity signal from lean and obese (a) AbdSc adipose tissue and (b) omental adipose tissue. Statistical analysis was performed using one-way ANOVA. White bars, secretory PLA2; light grey bars, cPLA2; dark grey bars, iPLA2; black bars, platelet-activating factor acetylhydrolase. $* p<0.05$
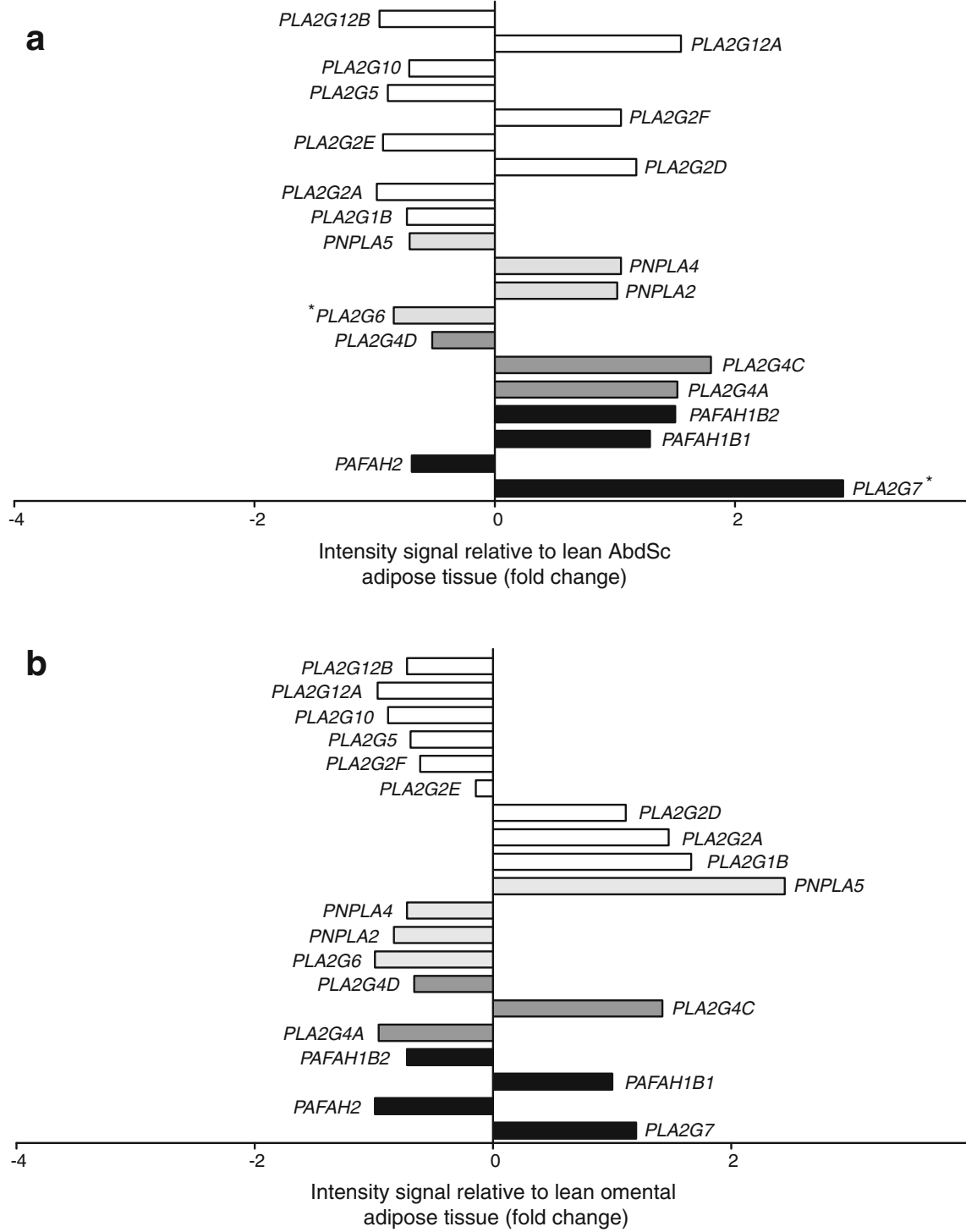

expression of these macrophage markers and PLA2G7 (see electronic supplementary material [ESM] Fig. 1a-e). Similarly, correlation analysis of EMR1 and PLA2G7 mRNA expression in AbdSc adipose tissue showed no significant correlation ( $r=0.192, p=0.530$; Fig. $4 \mathrm{~d}$ ). The results presented here clearly demonstrate the expression of Lp-PLA2 by adipocytes from human adipose tissue.

\section{Comparison of anthropometric and biochemical analytes in} subcohorts The association of Lp-PLA2 with selected metabolic markers was investigated to further understand the influence of metabolic states on circulating Lp-PLA2.

Table 1 shows baseline characteristics from fasted participants in four subcohorts. Obese participants and those with type 2 diabetes status had significantly higher levels of LpPLA2 compared with the lean group $(p<0.05)$; this association was attenuated in the overweight group. Compared with the lean study group, the obese and type 2 diabetic women also had statistically different levels of all other metabolic markers, apart from cholesterol in the obese group. These associations were not seen in the overweight participants, except for endotoxin levels, which were significantly increased $(p<0.05)$.

The relationships between circulating Lp-PLA2 and key metabolic markers were determined using linear regression. Analysis of our full cohort revealed that Lp-PLA2 positively correlated with cholesterol, LDL-cholesterol, oxLDL, BMI and endotoxin (Fig. 5). Subsequent subcohort analysis revealed significant positive correlations with metabolic markers, including cholesterol, triacylglycerol, LDL-cholesterol, LDL-cholesterol /HDL-cholesterol, endotoxin and oxLDL in non-diabetic individuals (Table 2). Additionally, strong correlations between Lp-PLA2 and HDL-cholesterol, LDL-cholesterol/HDL-cholesterol and oxLDL were maintained in individuals with type 2 diabetes.

Thereafter, multivariate stepwise regression analysis was used to identify factors that influence circulating Lp-PLA2 

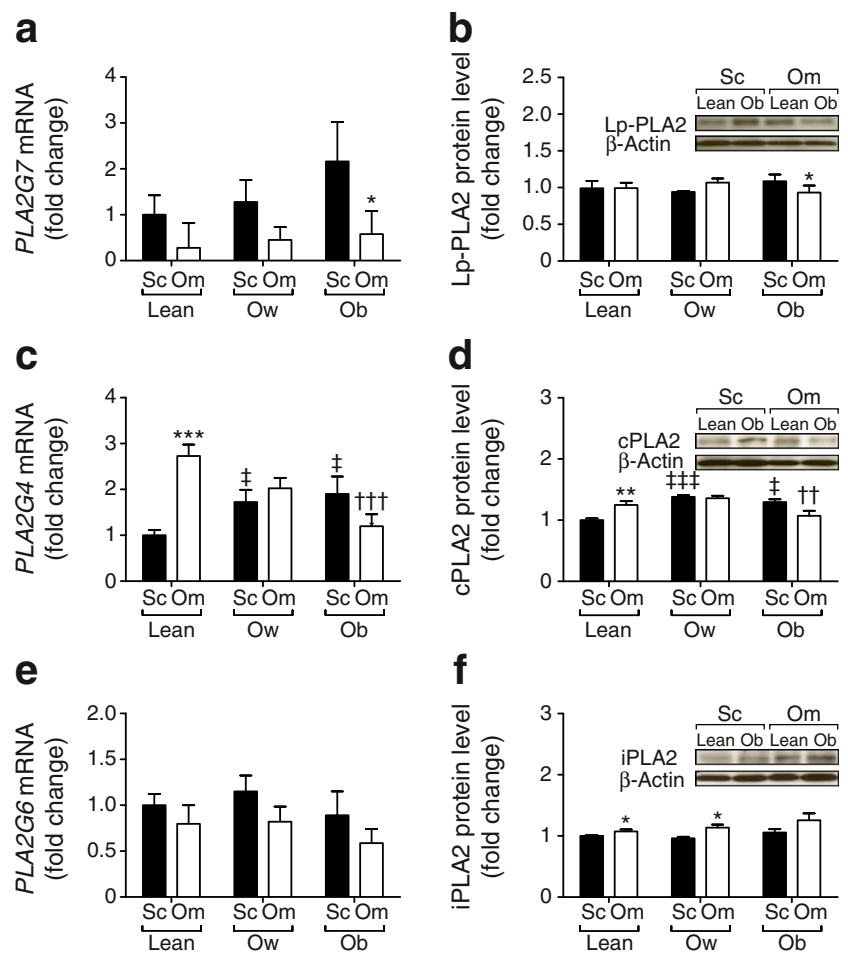

Fig. 2 mRNA and protein expression of PLA2 in AbdSc adipose tissue and omental adipose tissue from lean $(n=9)$, overweight $(n=10)$ and obese $(n=5)$ non-diabetic individuals. (a) PLA2G7 mRNA expression. (b) Lp-PLA2 protein levels. (c) PLA2G4 mRNA expression. (d) cPLA2 protein levels. (e) PLA2G6 mRNA expression. (f) iPLA2 protein levels. All qPCR and western blot results were standardised to lean AbdSc adipose tissue. Statistical analysis was performed using paired $t$ test. $* p<0.05, * * p<0.01$ and $* * * p<0.001$ for AbdSc vs omental adipose tissue; $\uparrow \dagger p<0.01$ and $\dagger \dagger \dagger p<0.001$ vs lean omental adipose tissue; and $\$ p<0.05$ and $\$ \uparrow t p<0.05$ vs lean AbdSc adipose tissue. Ob, obese; Om, omental adipose tissue; Ow, overweight; Sc, subcutaneous

across non-diabetic and diabetic groups. In the whole study population, LDL-cholesterol was the sole determinant of circulating Lp-PLA2, accounting for $9.7 \%$ of the variance observed (Table 3). Further analysis of type 2 diabetic individuals revealed that oxLDL, triacylglycerol and HDL-cholesterol
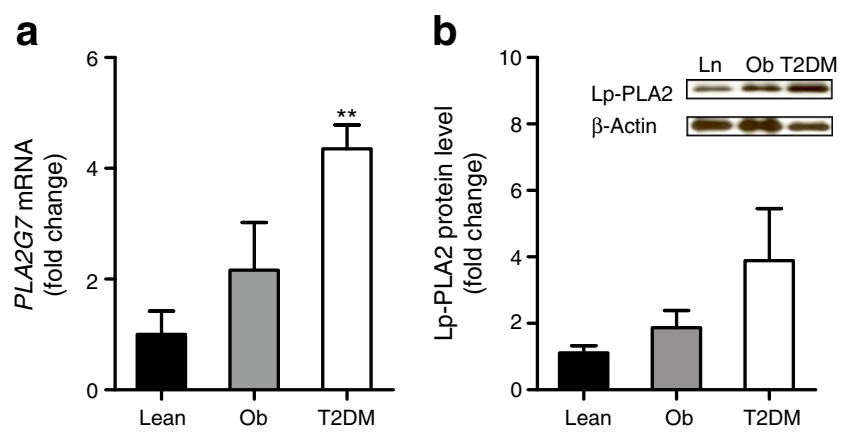

Fig. 3 Influence of type 2 diabetes status on mRNA expression and protein levels of Lp-PLA2. (a) PLA2G7 mRNA expression and (b) LpPLA2 protein expression in AbdSc adipose tissue in lean, obese and type 2 diabetic individuals. Statistical analysis was performed using paired $t$ tests. ${ }^{*} p<0.01$ vs lean AbdSc adipose tissue. Ln, lean; Ob, obese; T2DM, type 2 diabetes account for $59.7 \%$ of the variance $(p<0.001)$. In non-diabetic individuals, LDL-cholesterol was a significant predictor $(p=0.02)$, but not after the Bonferroni correction was applied.

These findings indicate that oxLDL, triacylglycerol and HDL-cholesterol are the most important predictors of LpPLA2 in patients with type 2 diabetes; additionally, correlation data show a clear link between lipid profile and Lp-PLA2 across different metabolic states.

Effect of Lp-PLA2 inhibitor, oxLDL and LDL-cholesterol on Chub-S7 cells Given that our study revealed an association between Lp-PLA2 and the unfavourable circulating lipid profile in type 2 diabetic individuals, we sought to determine the direct influence of lipid mediators on Lp-PLA2 activity.

Treatment of Chub-S7 cells with oxLDL induced an acute rise in PLA2G7 mRNA levels that was statistically significant at $6 \mathrm{~h}$ post treatment, and which slowly declined up until $48 \mathrm{~h}$ $(p<0.001$; Fig. 6a). Protein levels displayed similar results, with upregulation of Lp-PLA2 at $3 \mathrm{~h}$ and $6 \mathrm{~h}$, followed by a steep decline post $6 \mathrm{~h}$, although still significantly higher than the control $(p<0.001$, Fig. 6 b). Treatment with native LDLcholesterol resulted in increased PLA2G7 gene expression at $48 \mathrm{~h}$ compared with $3 \mathrm{~h}(p<0.001$; Fig. $6 \mathrm{c})$. However, $6 \mathrm{~h}$ stimulation with LDL-cholesterol diminishes PLA2G7 gene expression $(p<0.001$; Fig. $6 c)$. Interestingly, Lp-PLA2 protein levels increased at every time point post LDL-cholesterol treatment $(p<0.001$, Fig. $6 \mathrm{~d})$.

Further analysis of LDL treatment explored whether adipocytes were converting LDL into oxLDL, and how this conversion was affected by an Lp-PLA2 inhibitor. At $72 \mathrm{~h}$ incubation, the oxLDL level had significantly increased by $19.8 \%$ compared with $24 \mathrm{~h}$ incubation $(p<0.05)$ (Fig. 6e). The increased oxLDL production in medium significantly diminished when the cells were treated with LDL-cholesterol plus Lp-PLA2 inhibitor (Fig. 6e). Thus, these studies indicate a functional requirement for Lp-PLA2 in adipocytes for oxLDL production.

\section{Discussion}

The main purpose of this study was to evaluate the role of the adipocyte as a source of Lp-PLA2, and its capacity to influence oxLDL production as a contributing influence in inflammation within obesity-mediated diabetes. To establish this, we comprehensively evaluated PLA2 isoforms in different states of adiposity and diabetes status, noting the effect of cholesterol on cellular Lp-PLA2 in an in vitro adipocyte model. We report that PLA2 and its isoforms appear to be heavily influenced by weight, metabolic state and circulating lipids. Moreover, LpPLA2 is associated with an unfavourable circulating lipid profile, including increased oxLDL and triacylglycerol, which is exacerbated in type 2 diabetes. Furthermore, our in vitro adipocyte studies show that Lp-PLA2 is expressed and functional, 
Fig. 4 Analysis of Lp-PLA2 and macrophage markers in adipocytes and adipose tissue. (a) Representative images of immunohistochemical staining in human AbdSc adipose tissue and placenta, showing adipocytes expressing Lp-PLA2. (b) LpPLA2 protein levels in human primary culture and a human adipocyte cell line (Chub-S7) on day 0 and day 14 of differentiation. (c) Gene expression of PLA2G7 (LpPLA2) and macrophage markers in SVF and mature adipocytes. (d) Correlation between mRNA encoding the macrophagespecific marker EMR1, in AbdSc adipose tissue from type 2 diabetic individuals. The solid line represents linear regression. Statistical analysis was performed using two-way ANOVA (d) and paired $t$ test $(\mathbf{b}, \mathbf{c})$. Scale bar, $100 \mu \mathrm{m} . * * * p<0.001$. AT, adipose tissue; D, day; MA, mature adipocyte levels of PLA2G7 and EMR1,
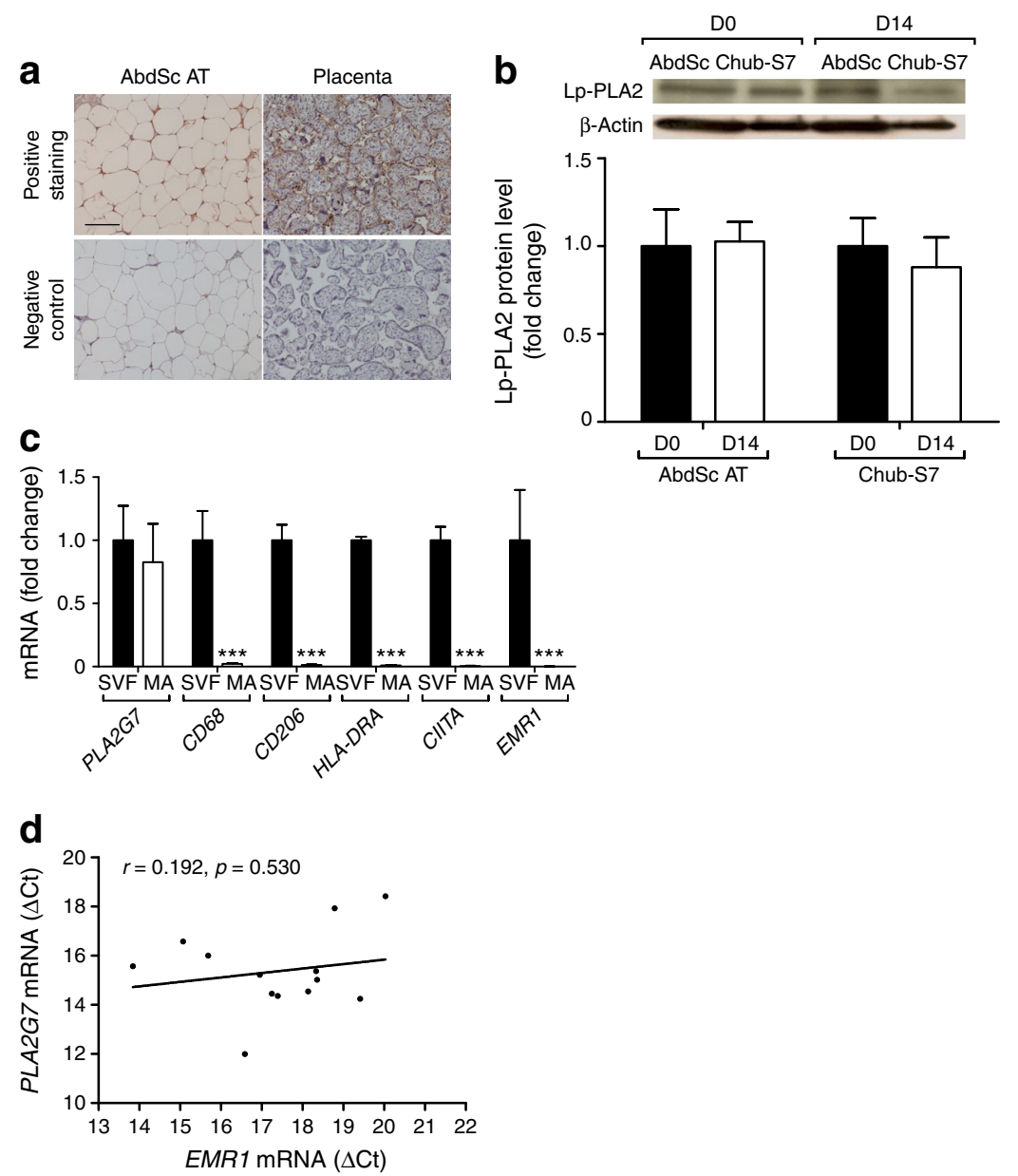

being influenced by LDL-cholesterol and oxLDL in a similar fashion to monocyte studies [21].

Obesity contributes to a heightened inflammatory response in adipose tissue [22]; the influence of the PLA2 superfamily in this condition has been unclear to date [23]. Its importance in adipose tissue may arise as cPLA2 is phosphorylated by c-Jun N-terminal kinase (JNK), p38 mitogen-activated protein kinase (MAPK) and p42/44 MAPKs - all known intracellular mediators of inflammation [24]. Similarly, iPLA2 and Lp-PLA2 are also considered pathogenic inflammatory markers in monocytes $[25,26]$. Our microarray analysis of PLA2 isoforms in AbdSc adipose tissue found that Lp-PLA2 and iPLA2 levels were altered by adiposity, but this was not mirrored by subsequent gene or protein expression data. However, cPLA 2 mRNA and protein levels increased with adiposity. The apparent disparity between our initial microarray results and subsequent mRNA and protein analysis may have reflected the limited number of participants available for microarray analysis. Furthermore, analysis of PLA2 isoforms in adipose tissue from a male cohort (given their higher metabolic risk for a given age and BMI) would add value to our existing knowledge.

It is clear from prior studies that macrophages represent an important source of Lp-PLA2 [27, 28], and increase with adiposity in omental adipose tissue [29, 30]. Therefore, it was important to ascertain the contribution of macrophages to LpPLA2 in adipose tissue, as well as to explore the potential cellular site of Lp-PLA2 expression. In brief, we: (1) evaluated the influence of macrophages, using the macrophage marker EMR1 to explore an association with Lp-PLA2 in adipose tissue [31, 32]; (2) analysed, immunohistochemically, the expression of Lp-PLA2 in adipose tissue; and (3) used isolated mature and differentiated human adipocyte cells to examine Lp-PLA2 and macrophage expression. These studies suggest that adipocytes can be viewed as an important contributing source of Lp-PLA2 expression in adipose tissue and adipocytes. Analysis of the mature adipocytes and adipocyte cell line highlighted that any perceived prior contamination of macrophages in cell cultures did not represent a source of Lp-PLA2 mRNA or protein expression. Therefore, targeting adipocytes in obese individuals with darapladib (an Lp-PLA2 inhibitor known to reduce plaque instability in coronary events [33, 34]) may effectively influence adipose tissue dysfunction in obese/type 2 diabetic individuals with and without CVD.

Prior studies have shown associations between Lp-PLA2 and CVD. Our studies examined circulating Lp-PLA2 and its relationship with selected metabolic markers in type 2 diabetic 
a
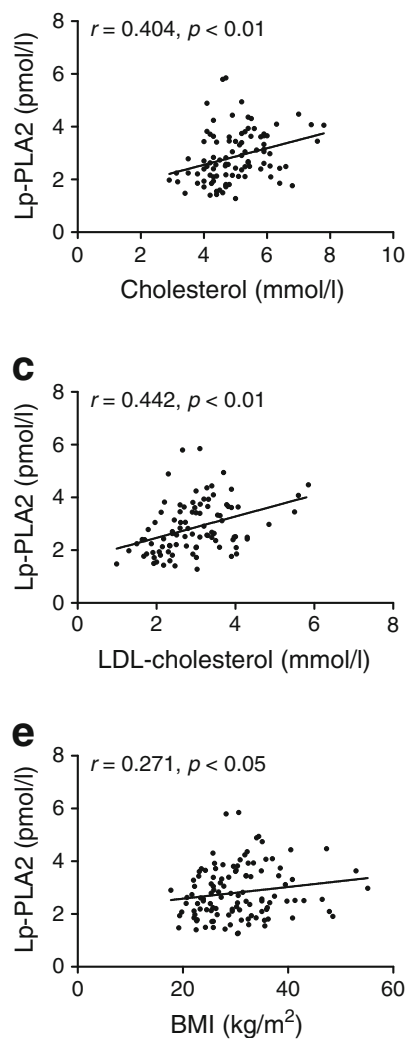

b
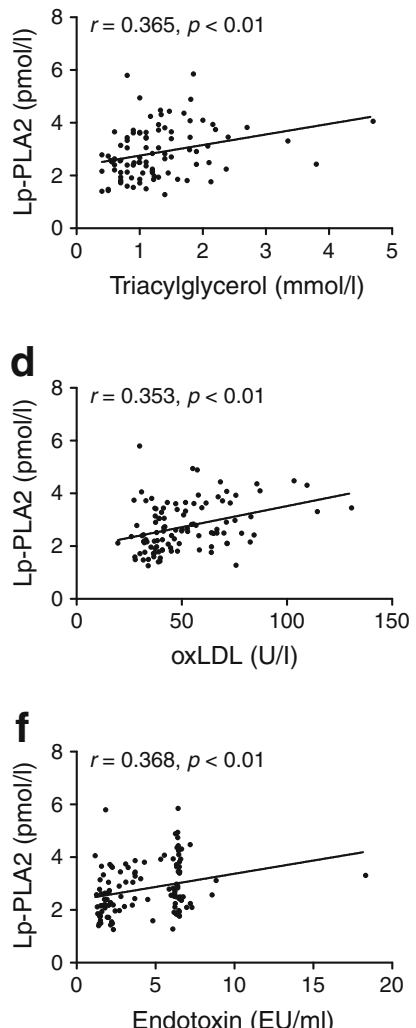

Fig. 5 Correlations between Lp-PLA2 (pmol/l) and: (a) cholesterol $(\mathrm{mmol} / \mathrm{l})$, (b) triacylglycerol (mmol/l), (c) LDL-cholesterol $(\mathrm{mmol} / \mathrm{l})$, (d) oxLDL (U/1), (e) BMI $\left(\mathrm{kg} / \mathrm{m}^{2}\right)$ and (f) endotoxin $(\mathrm{EU} / \mathrm{ml})$. Correlation analysis was performed using Spearman's rank correlation analysis, followed by two-way ANOVA

individuals. The data showed that circulating Lp-PLA2 increased significantly with both the level of adiposity and diabetes status. These findings support previous studies in women

with gestational diabetes [33] and South Asian individuals with the metabolic syndrome [34], which report similar increases in Lp-PLA2. Interestingly, relative to people without diabetes, there is a fourfold increase in intracellular Lp-PLA2 levels in adipose tissue from individuals with type 2 diabetes; this may, in part, explain the systemic changes in Lp-PLA2 plasma levels in type 2 diabetic individuals. It should also be noted that other cell types secrete Lp-PLA2, such as monocytes, macrophages, $\mathrm{T}$ lymphocytes and mast cells, providing an additional source of circulating Lp-PLA2 in type 2 diabetes [35, 36].

In common with people with CVD, the participants with type 2 diabetes in this study had a more unfavourable cardiometabolic risk profile than the obese individuals without diabetes; this included increased oxLDL and LDL-cholesterol/HDLcholesterol ratio and diminished HDL-cholesterol levels. Our multivariate stepwise regression analysis confirmed oxLDL, triacylglycerol and HDL-cholesterol as the most important predictors of Lp-PLA2 in participants with type 2 diabetes. Additionally, correlation data showed a clear link between lipid profiles and Lp-PLA2 across different metabolic states. These findings were partially affirmed by a study involving a cohort of men with type 2 diabetes, where a multiple-regression model found triacylglycerols as the key predictor of Lp-PLA2 [37]. Therefore, it appears that an unfavourable circulating lipid profile may drive an increase in Lp-PLA2 in adipose tissue, and this is more pronounced in individuals with type 2 diabetes.

The association of an unfavourable lipid profile with diabetes and the associated increase in intracellular Lp-PLA2 in adipose tissue in type 2 diabetes led us to investigate the importance of the adipocyte in LDL-cholesterol and oxLDL modulation in a human adipocyte cell system. In this cellular system, it was shown that acute oxLDL exposure increased the new synthesis of Lp-PLA2, as observed by increasing
Table 2 Correlations between serum Lp-PLA2 and selected metabolic markers

\begin{tabular}{llllll}
\hline Characteristic & \multicolumn{2}{l}{ Non-diabetic } & & \multicolumn{2}{l}{$\begin{array}{l}\text { Type 2 diabetic } \\
(n=35)\end{array}$} \\
\cline { 2 - 5 } & $\begin{array}{l}\text { Non-diabetic } \\
(n=79)\end{array}$ & $\begin{array}{l}\text { Lean } \\
(n=23)\end{array}$ & $\begin{array}{l}\text { Overweight } \\
(n=24)\end{array}$ & $\begin{array}{l}\text { Obese } \\
(n=32)\end{array}$ & \\
\hline BMI & $0.271^{*}$ & 0.146 & 0.113 & 0.138 & -0.042 \\
Glucose & 0.123 & 0.306 & 0.011 & 0.025 & 0.236 \\
HOMA-IR & 0.249 & 0.395 & 0.468 & 0.215 & 0.143 \\
Cholesterol & $0.404^{* *}$ & $0.537^{*}$ & 0.310 & 0.315 & 0.176 \\
Triacylglycerol & $0.365^{* *}$ & 0.459 & 0.288 & 0.299 & 0.146 \\
LDL-cholesterol & $0.442^{* *}$ & $0.607^{* *}$ & 0.221 & $0.400^{*}$ & 0.306 \\
HDL-cholesterol & $-0.281^{*}$ & 0.207 & 0.113 & $0.420^{*}$ & $-0.536^{* *}$ \\
LDL-cholesterol & $0.449^{* * *}$ & $0.596^{* *}$ & 0.025 & $0.486^{* *}$ & $0.588^{* * * *}$ \\
$\quad$ /HDL-cholesterol & $0.304^{* *}$ & 0.297 & 0.465 & 0.250 & 0.122 \\
Insulin & $0.368^{* *}$ & $0.590^{* *}$ & 0.125 & 0.326 & 0.071 \\
Endotoxin & $0.353^{* *}$ & $0.460^{*}$ & 0.196 & 0.328 & $0.657^{* * *}$ \\
OxLDL & & & & & \\
\hline
\end{tabular}

The values shown are Spearman's correlation coefficients $* p<0.05, * * p<0.01$ and $* * * p<0.001$ 
Table 3 Variables statistically associated with circulating LpPLA2 in multivariate stepwise regression analysis
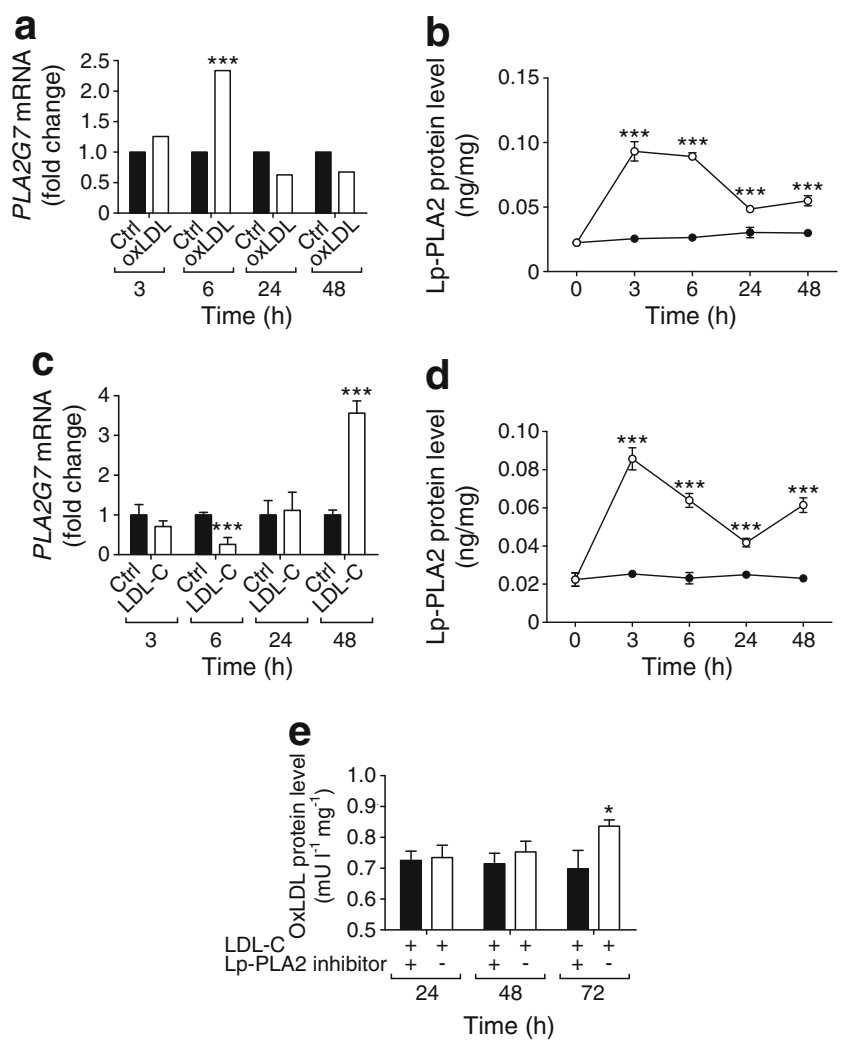

Fig. 6 Effects of an Lp-PLA2 inhibitor, oxLDL and LDL-cholesterol on Lp-PLA2 gene expression and protein levels. Differentiated Chub-S7 adipocytes $(n=6)$ were treated for 3, 6, 24 and $48 \mathrm{~h}$ with: (a) $43 \mathrm{pmol} / 1$ oxLDL, PLA2G 7 mRNA was measured; (b) 43 pmol/1 oxLDL, Lp-PLA2 protein levels were measured (black circles, control; white circles, oxLDL-treated cells); (c) 67 pmol/1 LDL-cholesterol, PLA2G7 mRNA was measured; and (d) $67 \mathrm{pmol} / 1$ LDL-cholesterol, Lp-PLA2 protein levels were measured (black circles, control; white circles, LDL-cholesterol-treated cells). (e) Cells were treated with $200 \mu \mathrm{g}$ protein/ml LDLcholesterol and with or without $20 \mu \mathrm{mol} / 1 \mathrm{Lp}-\mathrm{PLA} 2$ inhibitor for 24,48 and $72 \mathrm{~h}$. The oxLDL levels (mU/l) were normalised to individual total protein concentration. Statistical analysis was performed using two-way ANOVA. $* p<0.05$ and $* * * p<0.001$ vs $0 \mathrm{~h}$. Ctrl, control; LDL-C, LDLcholesterol gene expression. In comparison, oxLDL treatment in human THP-1 monocyte cell cultures also upregulated Lp-PLA2, suggesting that adipocytes may act in a similar manner to macrophages [21, 38]. Lp-PLA2 protein levels appeared to be induced at an even earlier time point than mRNA expression. This apparent disparity may have arisen because of the capacity of the adipocyte to influx and digest oxLDL and LDL-cholesterol, releasing fatty acid, cholesterol and LpPLA2 [39]. As such, Lp-PLA2 is transported into the adipocyte bound to apolipoprotein B on LDL-cholesterol, its primary carrier [40]. Therefore, the protein expression of LpPLA2 likely reflects the exogenous Lp-PLA2 available, while the mRNA expression represents the newly synthesised LpPLA2. Of note, LDL-cholesterol treatment led to similar findings for Lp-PLA2 protein levels: following acute treatment we observed elevated levels of Lp-PLA2, with a gradual reduction over time. To further implicate Lp-PLA2 in the regulation of lipid mediators, co-treatment of LDL-cholesterol with an Lp-PLA2 inhibitor was tested, resulting in diminished oxLDL-mediated inflammation. Thus, Lp-PLA2 functionality within adipocytes may be required for oxLDL production, and the ability to modify oxLDL production from adipose tissue through the Lp-PLA2 pathway may be an important mechanism to target. Future knockdown studies of Lp-PLA2 could further evaluate these effects on adipocyte cellular functions. This may also ascertain whether adipocyte Lp-PLA2 is a useful therapeutic target to improve the lipid profile of people with type 2 diabetes. While still appreciating that downregulating Lp-PLA2 in adipocytes may be significant in reducing systemic triacylglycerols and oxLDL levels, it should be stressed that, as lipid control is a multifactorial process, examining more than one mediator would enhance our understanding of the potential impact on lipid profiles.

In conclusion, this work has highlighted that AbdSc adipose tissue and the adipocyte may act as separate and significant sources of oxLDL production (i.e. separate from foam cells 
within unstable atherogenic plaques noted in coronary artery disease) [41]. Furthermore, human adipose tissue and adipocytes appear active sources of Lp-PLA2, with expression induced by LDL-cholesterol and oxLDL. Lp-PLA2 expression is raised in $\mathrm{AbdSc}$ adipose tissue from people without diabetes, and this is further enhanced in the type 2 diabetic state. The observed increase of Lp-PLA2 in type 2 diabetic people appears to be associated with an upregulation in systemic lipids. As such, increased Lp-PLA2 protein from adipocytes in obesity and type 2 diabetes may contribute to increased circulating oxLDL levels. In turn, this may further promote inflammation and increase the atherosclerotic risk. Therefore, Lp-PLA2 action within adipocytes appears to represent a novel and important therapeutic target to reduce inflammation, atherosclerotic risk and the development of cardiometabolic complications in type 2 diabetes.

Acknowledgements We extend our thanks to the surgeons, theatre staff and Arden Tissue Bank at University Hospitals Coventry and Warwickshire NHS Trust Hospital, Coventry for the collection of samples. We also acknowledge S. L. Sabico (King Saud University, Riyadh, Saudi Arabia) and P. D. Voyias (University of Warwick, Coventry, UK) for their contributions to the paper.

Funding LJ was a recipient of the Warwick Medical School Chancellors $\mathrm{PhD}$ Scholarship. WK was supported by the Thai Government during her doctoral studies. This work was also partly funded by supporting grants from the Rowlands Trust and Coventry \& District Charitable Trust.

Data availability Any data not included within this paper are available from the corresponding author on reasonable request.

Duality of interest The authors declare that there is no duality of interest associated with this manuscript.

Contribution statement $\mathrm{LJ}$ and WK contributed to the acquisition, analysis and interpretation of data, preparation of figures and writing of the manuscript. JDM implemented, managed and performed the microarray analysis. NA, IK and TMB contributed to the data collection, its analysis and intellectual input for revision of the manuscript. HR and SK contributed to the analysis and interpretation of the data. GT and PGM led the design of the study, analysis and interpretation of the data and manuscript writing and revision. PGM led the grant applications to source the funding of the studies. All authors corrected, revised and gave their approval for the final manuscript to be published. GT and PGM are the guarantors of this work.

Open Access This article is distributed under the terms of the Creative Commons Attribution 4.0 International License (http:// creativecommons.org/licenses/by/4.0/), which permits unrestricted use, distribution, and reproduction in any medium, provided you give appropriate credit to the original author(s) and the source, provide a link to the Creative Commons license, and indicate if changes were made.

\section{References}

1. Zalewski A, Nelson JJ, Hegg L, Macphee C (2006) Lp-PLA2: a new kid on the block. Clin Chem 52:1645-1650
2. Rosenson RS, Stafforini DM (2012) Modulation of oxidative stress, inflammation, and atherosclerosis by lipoprotein-associated phospholipase A2. J Lipid Res 53:1767-1782

3. Toth PP, McCullough PA, Wegner MS, Colley KJ (2010) Lipoprotein-associated phospholipase A2: role in atherosclerosis and utility as a cardiovascular biomarker. Expert Rev Cardiovasc Ther 8:425-438

4. Oei HH, Van Der Meer IM, Hofman A et al (2005) Lipoproteinassociated phospholipase $\mathrm{A} 2$ activity is associated with risk of coronary heart disease and ischemic stroke: the Rotterdam Study. Circulation 111:570-575

5. Winkler K, Hoffmann MM, Winkelmann BR et al (2007) Lipoprotein-associated phospholipase A2 predicts 5-year cardiac mortality independently of established risk factors and adds prognostic information in patients with low and medium highsensitivity C-reactive protein (the Ludwigshafen risk and cardiovascular health study). Clin Chem 53:1440-1447

6. Khakpour H, Frishman WH (2009) Lipoprotein-associated phospholipase A2: an independent predictor of cardiovascular risk and a novel target for immunomodulation therapy. Cardiol Rev 17:222-229

7. Kinney GL, Snell-Bergeon JK, Maahs DM et al (2011) Lipoprotein-associated phospholipase A2 activity predicts progression of subclinical coronary atherosclerosis. Diabetes Technol Ther 13:381-387

8. Vickers KC, Maguire CT, Wolfert R et al (2009) Relationship of lipoprotein-associated phospholipase A2 and oxidized low density lipoprotein in carotid atherosclerosis. J Lipid Res 50:1735-1743

9. Waegner AM, Sanchez-Quesada LJ, Benitez S, Bancells C, Ordonez-Llanos J, Perez A (2011) Effect of statin and fibrate treatment on inflammation in type 2 diabetes. A randomized, cross-over study. Diabetes Res Clin Pract 93:E25-E28

10. Nelson TL, Kamineni A, Psaty B et al (2011) Lipoproteinassociated phospholipase A2 and future risk of subclinical disease and cardiovascular events in individuals with type 2 diabetes: the Cardiovascular Health Study. Diabetologia 54:329-333

11. Nakos G, Kitsiouli E, Hatzidaki E, Koulouras V, Touqui L, Lekka ME (2005) Phospholipases A(2) and platelet-activating-factor acetylhydrolase in patients with acute respiratory distress syndrome. Crit Care Med 33:772-779

12. Schipper HS, Nuboer R, Prop S et al (2012) Systemic inflammation in childhood obesity: circulating inflammatory mediators and activated CD14++ monocytes. Diabetologia 55:2800-2810

13. Creely SJ, McTernan PG, Kusminski CM et al (2007) Lipopolysaccharide activates an innate immune system response in human adipose tissue in obesity and type 2 diabetes. Am J Physiol Endocrinol Metab 292:740-747

14. Harte AL, Varma MC, Tripathi G et al (2012) High fat intake leads to acute postprandial exposure to circulating endotoxin in type 2 diabetic subjects. Diabetes Care 35:375-382

15. McTernan P, Anwar A, Eggo M, Barnett A, Stewart P, Kumar S (2000) Gender differences in the regulation of P450 aromatase expression and activity in human adipose tissue. Int $\mathrm{J}$ Obes Relat Metab Disord 24:875-881

16. Bradford MM (1976) A rapid and sensitive method for the quantitation of microgram quantities of protein utilizing the principle of protein-dye binding. Anal Biochem 72:248-254

17. Towbin H, Staehelin T, Gordon J (1979) Electrophoretic transfer of proteins from polyacrylamide gels to nitrocellulose sheets: procedure and some applications. Proc Natl Acad Sci U S A 76:4350-4354

18. Saiki A, Olsson M, Jernas M et al (2009) Tenomodulin is highly expressed in adipose tissue, increased in obesity, and downregulated during diet-induced weight loss. J Clin Endocrinol Metab 94:3987-3994

19. Anveden A, Sjoholm K, Jacobson P et al (2012) ITIH-5 expression in human adipose tissue is increased in obesity. Obesity 20:708-714 
20. Darimont C, Zbinden I, Avanti O et al (2003) Reconstitution of telomerase activity combined with HPV-E7 expression allow human preadipocytes to preserve their differentiation capacity after immortalization. Cell Death Differ 10:1025-1031

21. Wang WY, Li J, Yang D, Xu W, Zha RP, Wang YP (2010) OxLDL stimulates lipoprotein-associated phospholipase A2 expression in THP-1 monocytes via PI3K and p38 MAPK pathways. Cardiovasc Res 85:845-852

22. van Greevenbroek M, Schalkwijk C, Stehouwer C (2013) Obesityassociated low-grade inflammation in type 2 diabetes mellitus: causes and consequences. Neth J Med 71:174-187

23. Shi Y, Zhang P, Zhang L et al (2007) Role of lipoprotein-associated phospholipase A2 in leukocyte activation and inflammatory responses. Atherosclerosis 191:54-62

24. Wang X, Xue H, Xu Q et al (2008) p38 kinase/cytosolic phospholipase A2/cyclooxygenase-2 pathway: a new signaling cascade for lipopolysaccharide-induced interleukin-1 $\beta$ and interleukin- 6 release in differentiated U937 cells. Prostaglandins Other Lipid Mediat 86:61-67

25. Xie Z, Gong MC, Su W, Xie D, Turk J, Guo Z (2010) Role of calcium-independent phospholipase A2beta in high glucoseinduced activation of RhoA, Rho kinase, and CPI-17 in cultured vascular smooth muscle cells and vascular smooth muscle hypercontractility in diabetic animals. J Biol Chem 285:8628-8638

26. Piñón P, Kaski J (2006) Inflammation, atherosclerosis and cardiovascular disease risk: PAPP-A, Lp-PLA2 and cystatin C. New insights or redundant information? Rev Esp Cardiol 59:247-258

27. Sudhir K (2005) Lipoprotein-associated phospholipase A2, a novel inflammatory biomarker and independent risk predictor for cardiovascular disease. J Clin Endocrinol Metab 90:3100-3105

28. McConnell JP, Jaffe AS (2009) The spin stops here: inhibition of lipoprotein-associated phospholipase $\mathrm{A} 2$ - a promising target but a negative initial trial? Clin Chem 55:21-23

29. Weisberg S, McCann D, Desai M, Rosenbaum M, Leibel R, Ferrante A (2003) Obesity is associated with macrophage accumulation in adipose tissue. J Clin Invest 112:1796-1808

30. Boutens L, Stienstra R (2016) Adipose tissue macrophages: going off track during obesity. Diabetologia 59:879-894
31. Fink LN, Oberbach A, Costford SR et al (2013) Expression of antiinflammatory macrophage genes within skeletal muscle correlates with insulin sensitivity in human obesity and type 2 diabetes. Diabetologia 56:1623-1628

32. Harford KA, Reynolds CM, McGillicuddy FC, Roche HM (2011) Fats, inflammation and insulin resistance: insights to the role of macrophage and $\mathrm{T}$ cell accumulation in adipose tissue. Proc Nutr Soc 70:408-417

33. Wilensky RL, Shi Y, Mohler ER et al (2008) Inhibition of lipoprotein-associated phospholipase A2 reduces complex coronary atherosclerotic plaque development. Nat Med 14:1059-1066

34. White H (2010) Darapladib and its potential for plaque stabilization and prevention of cardiac events. Clin Lipidol 5:465-476

35. Ulrich C, Trojanowicz B, Fiedler R et al (2017) Differential expression of lipoprotein-associated phospholipase A2 in monocyte subsets: impact of uremia and atherosclerosis. Nephron 135:231-241

36. Persson M, Nilsson J, Nelson JJ, Hedblad B, Berglund G (2007) The epidemiology of Lp-PLA(2): distribution and correlation with cardiovascular risk factors in a population-based cohort. Atherosclerosis 190:388-396

37. Noto H, Chitkara P, Raskin P (2006) The role of lipoproteinassociated phospholipase A2 in the metabolic syndrome and diabetes. J Diabetes Complicat 20:343-348

38. Yang M, Chu EM, Caslake MJ, Edelstein C, Scanu AM, Hill JS (2010) Lipoprotein-associated phospholipase A2 decreases oxidized lipoprotein cellular association by human macrophages and hepatocytes. Biochim Biophys Acta 1801:176-182

39. Mentese A, Sumer A, Sumer A, Demir S (2016) Effects of homocysteine on adipocyte differentiation and CD36 gene expression in 3T3-L1 adipocytes. J Cell Commun Signal 10:55-60

40. Zalewski A, Macphee C (2005) Role of lipoprotein-associated phospholipase A2 in atherosclerosis: biology, epidemiology, and possible therapeutic target. Arterioscler Thromb Vasc Biol 25: 923-931

41. Johnson JL, Shi Y, Snipes R et al (2014) Effect of darapladib treatment on endarterectomy carotid plaque lipoproteinassociated phospholipase A2 activity: a randomized, controlled trial. PLoS One 9:e89034 